\title{
Molecular insights into the benefits of regular exercise in combating immunological mayhem during SARS-CoV-2 infection
}

Deepyaman Das ( $\sim$ deepyaman.das@gmail.com )

Raiganj University

\section{Research Article}

Keywords: exomiRs, Differentially expressed genes (DEGs), miRNA-mRNA interactions, RNA-seq, SARSCoV-2, Exercise

Posted Date: August 26th, 2021

DOl: https://doi.org/10.21203/rs.3.rs-845977/v1

License: (a) (i) This work is licensed under a Creative Commons Attribution 4.0 International License.

Read Full License 


\section{Abstract}

COVID-19 has caused devastation around the world with death tolls increasing every day. Case studies and hospital data suggest that immunocompromised individuals are at a greater risk of serious consequences caused by this disease. Regular exercise has been proven to combat multifarious diseases by improving overall health of the individual. A recent study has proven that regular exercise can reduce the serious illness associated with COVID-19. Circulating miRNAs released from the muscles during strenuous exercise has also been found to have anti-inflammatory effects. So, it was hypothesized that regular exercise might be releasing therapeutic miRNAs in the blood that might be reducing the immunological chaos in COVID-19 patients. Using network and systems biology approach, mRNA targets of 3 upregulated exomiRs (hsa-miR-486-5p, hsa-miR-215-5p, hsa-miR-941) in the blood of regularly exercising adults were mapped in the blood of COVID-19 patients. hsa-miR-215-5p, hsa-miR-486-5p and hsa-miR-941 were found to target 8,93 and 99 upregulated mRNAs respectively. Functional enrichment analysis showed that hsa-miR-486-5p might be preventing thrombosis and aggravated inflammation in regularly exercising COVID-19 patients. Thus, hsa-miR-486-5p can be considered to have therapeutic roles against immunological damage caused by COVID-19.

\section{Highlights}

- MIR215, MIR486 and MIR941 are upregulated exomiRs in blood of trained adults.

- MIR215, MIR486 and MIR941 might target 8, 93 and 99 mRNAs in COVID-19 blood.

- MIR486 might be a natural therapeutic agent against SARS-CoV-2.

\section{Introduction}

Increasing cases of COVID-19 (Corona virus disease 2019) and its recurring waves have compelled us to stay indoors. With the imposition of lockdown and social distancing measures, sedentary lifestyle has become the new normal. Sedentary lifestyle paves the pathway for various comorbid lifestyle diseases like cardiovascular diseases and diabetes that increases the complicacies associated with the disease. Moderate forms of exercise has been also proven to boost immunity (Nieman and Wentz, 2019). Experiments on influenza infected respiratory tract of animal models have shown that moderate exercise reduced mortality associated with the diseases (Lowder et al., 2005; Warren et al., 2015). Not only does physical activity reduce the chances of comorbid diseases but also it has been proven to reduce the fatality caused due to COVID-19 infection (Sallis et al., 2021). It was found that physical activity reduces the chances of both admissions to ICU and death. WHO (World Health organization) also recommends 150 min of moderate intensity or 75 min of intense exercise per week during the quarantine period. So, studying the molecular mechanism behind benefits of exercise would unravel natural therapeutic measures against the disease.

Regular exercise was found to release circulating miRNAs that help in healing the body after a strenuous exercise (Sapp et al., 2017). These circulating miRNAs has anti-inflammatory roles (Gomes et al., 2015). 
ExomiRNAs (exomiRs) are exosomes loaded with miRNAs that are released from various cells into the blood and they mediate cellular crosstalk by acting as intercellular messaging system (Valadi et al., 2007). So, it was hypothesized that exomiRs in the blood of regularly exercising adults might be having therapeutic roles against inflammatory rage of COVID-19. By using computational and systems biology approach, 3 upregulated miRNAs from the blood of regularly exercising were screened and their targets were mapped in the blood of COVID-19. After functional analysis it was found that the miRNA hsa-miR486-5p might be a key player that is aiding to dampen the chances of thrombosis and aggravated inflammation in the blood of regularly exercising adults when infected with SARS-CoV-2.

\section{Materials And Methods}

\subsection{Data processing to screen exomiRs and DEGs from RNA-seq datasets}

The exomiRs were identified in GSE144627 (Nair et al., 2020) dataset. Here, the authors considered 5 sedentary males and 5 age-matched trained males for the study. The circulating exomiRs were identified by comparing their baseline expression profiles in the blood. Similar to the authors, DESeq2 (Love et al., 2014)in R v4.1.0 with a cut off of $|\log 2 \mathrm{FC}|>1.0$ and Wald test $p$-value $<0.05$ was considered for determining exomiRs.

For screening upregulated genes in the blood of COVID-19 patients two datasets- GSE152418 (Arunachalam et al., 2020) and GSE171110 (Lévy et al., 2021) were used. In GSE152418 (Arunachalam et al., 2020), the authors performed RNA-seq of PBMCs from the blood of 17 COVID-19 subjects and 17 age and sex- matched controls. In GSE171110 (Lévy et al., 2021), the authors considered whole blood transcriptome of 44 COVID-19 patients and 10 healthy donors. For both these studies, gene specific raw counts were analyzed using DESeq2 (Love et al., 2014) package in R v4.1.0 and DEGs were identified by considering a cut off of $\left|\log _{2} \mathrm{FC}\right|>1.0$ and adj. $\mathrm{p}$-value $<0.05$. Next, only the common upregulated genes from both the studies i.e. having $\log _{2} \mathrm{FC}>1$ were considered for meta-analysis in metaRNAseq v1.0.5 (Rau et al., 2014) in R v4.1.0. Among the two p-value combination techniques used by metaRNAseq v1.0.5 (Rau et al., 2014), inverse normal method was used to integrate these datasets.

\subsection{Retrieval of exomiRNA-mRNA interactions}

To determine the exomiRNA- mRNA interactions, miRWalk v3 (Sticht et al., 2018) was used by considering a high binding probability (binding prob. $=1$ ). The network was then visualized in Cytoscape 3.8.0 (Shannon et al., 2003).

\subsection{GO Biological Process enrichment of the mRNAs}

For getting functional insights of the mRNAs controlled by the miRNAs, GO biological process overrepresentation (enrichment) was done using clusterProfiler v4.0.0 (Yu et al., 2012) in R v4.1.0 by considering an adj. $P$ value $<0.05$. 


\section{Results}

\subsection{Identification of circulating DE miRNAs in the blood of regularly exercising adults}

Circulating exomiRs has been proposed to have multifarious roles in modulating post-transcriptional gene expression of different tissues by binding to 3'UTR of transcripts (Gorji-Bahri et al., 2018). Also exomiRs have been identified to modulate innate immune response (Li et al., 2006). Regular exercise not only boosts immunity but also has anti-inflammatory role against chronic inflammation (Gleeson et al., 2011).So, circulating miRNAs in the blood of regularly exercising adults might have therapeutic roles against inflammatory response during SARS-CoV-2 infection. To analyze the effect of exercise in SARSCoV-2 patients, initially a publicly available dataset - GSE144627 (Nair et al., 2020) was used to identify circulating exomiRs at rest in the blood of regularly exercising adults (trained). Using DESeq2 (Love et al., 2014), 4 DE miRNA were identified by considering a cut off of $|\log 2 \mathrm{FC}|>1.0$ and Wald test $p$-value $<0.05$. Among these, 3 miRNAs (hsa-miR-486-5p, hsa-miR-215-5p, hsa-miR-941) were found to be upregulated that were considered for downstream analysis (Supplementary file S1).

\subsection{Identification of DEGs in the blood cells of COVID-19 patients}

For identifying DE miRNAs, two blood transcriptome of SARS-CoV-2 infected patients- GSE152418 (Arunachalam et al., 2020) and GSE171110 (Lévy et al., 2021) were used. By using DESeq2 (Love et al., 2014) in R v4.1.0, 4151 (Fig. 1A) and 4475 DEGs (Fig. 1B) in GSE171110 (Lévy et al., 2021) and GSE152418 (Arunachalam et al., 2020) were identified respectively. DEG selection was done considering a cut off of $|\log 2 \mathrm{FC}|>1.0$ and adj. P value $<0.05$. Among these DEGs, only $1081 \mathrm{common}$ upregulated genes (Fig. 1C and 1D) (Supplementary File S1) from both the datasets were meta-analyzed by using metaRNAseq v1.0.5 (Rau et al., 2014) in R v4.1.0 and considered for downstream analysis.

\subsection{Reconstruction exomiRNA-mRNA network in blood of COVID-19 patients}

It was hypothesized that the upregulated exomiRNAs in blood of regularly exercising adults might be safeguarding them from the severity caused due to COVID-19 by translational silencing of upregulated genes in blood cells. To address this hypothesis, exomiRNA - mRNA interactions from miRWalk v3 (Sticht et al., 2018) was used and the network was visualized in Cytoscape 3.8.0 (Shannon et al., 2003). The network consists of 180 nodes and 200 edges (Fig. 2). hsa-miR-215-5p, hsa-miR-486-5p and hsamiR-941 were found to interact with 8, 93 and 99 upregulated mRNAs respectively.

3.4. Functional enrichment of the interacting mRNAs to gain insights into processes being dysregulated by the exomiRs. 
Then, GO Biological Process enrichment of the interacting mRNAs for each exomiR was performed using clusterProfiler v4.0.0 (Yu et al., 2012) in R v4.1.0 (Supplementary File S2) by considering a cut off of adj. $P$ value $<0.05$. Based on functional enrichment results, hsa-miR-486-5p and hsa-miR-941 were found to regulate platelet degranulation and mitotic cell division respectively (Fig. $3 \mathrm{~A}$ and $3 \mathrm{~B}$ ).

\section{Discussion}

During the recent COVID-19 outbreak, it was found that blood coagulation factors are altered as a result of infection (Tang et al., 2020). It causes moderate to severe thrombocytopenia along with increased Ddimer levels. Blood coagulation is the fastest and ultimate response to infections. Any wound in the body causes activation of endothelial cells and thereby leads to activation of prothrombin as a result of which blood coagulation is initiated (Henry et al., 2020). When the immune system is hyperactivated, coagulation might happen within the blood vessel leading to disseminated intravascular coagulation (DIC) (Minasyan and Flachsbart, 2019). DIC causes multiple organ failure. Strikingly, it has already been reported that COVID-19 causes altered coagulation and also organ dysfunction(Tang et al., 2020). Apart from triggering inflammation, the cytokine storm induced by SARS-CoV-2 also leads to increased fibrin deposition thereby causing hypercoagulation (Vivas et al., 2020). Again this inflammation causes activation of polymorphonuclear leukocytes (PMNs). PMNs inactivate endogenous anticoagulants and thereby leads to the formation of blood clots within blood vessels (Henry et al., 2020).

Sedentary lifestyle paves the pathway for multifarious diseases among which obesity and type 2 diabetes (T2DM) can cause a havoc (Park et al., 2020). T2DM can damage blood vessels and thereby magnify hypercoagulation in COVID-19 patients (Ayres, 2020). Moreover, obesity might aggravate overactivity of coagulation factors due to dysregulation of adipokines (Ryan and Caplice, 2020). Regular exercise has not only been proven to boost immunity but also triggers anti-inflammatory pathways (Gleeson et al., 2011). This helps in preventing severity caused due to systemic inflammation. Moreover, regular exercise reduces the risk of lifestyle diseases and reduces ill effects of adipokines (Gleeson et al., 2011). It was also reported that regular exercise might decrease platelet aggregation and fibrinolysis over time (Womack et al., 2003). So, patients having an active lifestyle might be able to combat immunogenic mayhem in COVID-19 by preventing thrombosis and hypercoagulation issues.

Discovery of exomiRs has helped in exploring previously unknown vistas of molecular biology (Bhome et al., 2018). ExomiRs are exosomes loaded with microRNAs that help in cellular crosstalk. Previous literature suggests that regular exercise causes the release of circulating miRNAs from injured tissues into the blood (Gomes et al., 2015). Again circulating miRNAs have been proven to have antiinflammatory roles (Tahamtan et al., 2018). As regular exercise has anti-inflammatory roles too, so an active lifestyle might be releasing circulating miRNAs in the blood that might be combating the aggravated inflammatory response caused by COVID- 19 .

For this, initially 3 upregulated exomiRNAs - hsa-miR-486-5p, hsa-miR-215-5p and hsa-miR-941 (Supplementary File S1) were identified from the blood of regularly exercising adults. hsa-miR-486-5p and 
hsa-miR-215-5p have roles in modulating the immune system. hsa-miR-486-5p has immunosuppressive roles as it inhibits IL-22 in cancer (Li et al., 2019). hsa-miR-215-5p was previously reported to suppress inflammation mediated injury (Yao et al., 2020). As it was hypothesized that exomiRNAs might be downregulating expression of genes within blood cells, so 1081 upregulated genes from the blood of COVID-19 infected patients was considered for mapping the targets of miRNAs (Fig. 1C and 1D) (Supplementary File S1). Important upregulated genes included - PCSK9 and IFI27. PCSK9 alters serum cholesterol levels and inhibitors of this gene has already been proposed as a therapeutic measure against SARS-CoV-2 (Vuorio and Kovanen, 2021). Again IFI27 has been proposed as blood biomarker for COVID19 (Gao et al., 2021). Mapping of target mRNA of the miRNAs using miRWalk v3 (Sticht et al., 2018) revealed that hsa-miR-215-5p, hsa-miR-486-5p and hsa-miR-941 targeted 8, 93 and 99 upregulated mRNAs. Some of important targets of hsa-miR-486-5p in this study are- FSTL1 and CX3CL1. FSTL1 was earlier reported to induce inflammation by triggering release of pro-inflammatory cytokines (Chaly et al., 2014). Whereas, CX3CL1 is a chemokine that leads to COVID-19 -associated thrombosis (Rivas-Fuentes et al., 2021). hsa-miR-941 was found to target cell cycle regulator CDK1. It has been reported that CDK1 induces IFN1 production in response to viral infection (Cingöz and Goff, 2018). Thus, the exomiRNAs might be targeting key players of the immune response and thereby reducing SARS-CoV-2 mediated hyperinflammation. To gain functional insights into the processes deregulated by these miRNAs, GO biological process enrichment was performed. It was found that hsa-miR-486-5p targeted platelet degranulation and leukocyte cell migration. It was reported that platelet activation and degranulation is a pathophysiological feature of COVID-19 (Zaid et al., 2020). Moreover, this also contributes to release of inflammatory cytokines that might magnify the cytokine storm in these patients. Important targets enriched for this GO function include - IGF1 and ITGB3 (Fig. 4). IGF1 was found to escalate platelet activation through IRS/PI3Kalpha pathway (Hers, 2007). Whereas, ITGB3 was found to modulate serotonin transport and thereby platelet aggregation in mice (Carneiro et al., 2008). Platelet degranulation has been reported to recruit neutrophils at the site of inflammation via release of serotonin (Duerschmied et al., 2013). So, migration of neutrophil like leukocytes occurs concomitantly with platelet aggregation. Some important mRNAs enriched in the GO biological process- "Leukocyte migration involved in inflammatory response" that are targeted by hsa-miR-486-5p include - FFAR2 and CX3CL1 (Fig. 4). Both FFAR2 and CX3CL1 were found to facilitate neutrophil migration to the site of inflammation (Björkman et al., 2016; Jones et al., 2010). These results suggest that hsa-miR-486-5p might be preventing platelet degranulation and by this process is also preventing leukocytes to be migrate to the site of inflammation. Thus, regular moderate forms of exercise might increase circulating hsa-miR-486-5p that might dampen the mayhem by reducing blood coagulation and leucocyte migration if infected with SARS-CoV-2 in the future. Apart from this, hsa-miR-486-5p might also be considered as a therapeutic measure against the inflammation caused by COVID-19 infection.

\section{Conclusion}

COVID-19 has brought life to a standstill. Although vaccines and steroids have been effective in reducing the mortality due to this disease, but boosting personal immunity have been proven to reduce serious 
health issues associated with this disease. The Medical fraternity is constantly advising to have an active lifestyle during this period. So, studying the therapeutic perspective of regular exercise in reducing aggravated immune response in COVID-19 needs attention. In this study, circulating miRNAs (exomiRs) in the blood of regularly exercising adults were screened and their targets were identified among the upregulated mRNAs in the blood of COVID-19 patients. The results suggest that hsa-miR-486-5p might be reducing the chances of thrombosis and aggravated inflammation in the blood of regularly exercising adults when infected with SARS-CoV-2. Further experimental studies need to be carried out to establish its therapeutic role against COVID-19.

\section{Declarations}

\section{Author contribution}

DD designed the study, devised the methodology, performed the analysis and wrote the manuscript.

\section{Acknowledgements}

DD is thankful to Raiganj University for providing the infrastructure for this work. He is also grateful for SVMCM (Swami Vivekananda Merit-Cum-Means) fellowship by the Government of West Bengal, India.

\section{Declaration of Competing Interest}

The author declares that there isn't any conflict of interest with any organization or financial entity in private or in public.

\section{References}

Arunachalam, P.S., Wimmers, F., Mok, C.K.P., Perera, R.A.P.M., Scott, M., Hagan, T., Sigal, N., Feng, Y., Bristow, L., Tak-Yin Tsang, O., Wagh, D., Coller, J., Pellegrini, K.L., Kazmin, D., Alaaeddine, G., Leung, W.S., Chan, J.M.C., Chik, T.S.H., Choi, C.Y.C., Huerta, C., Paine McCullough, M., Lv, H., Anderson, E., Edupuganti, S., Upadhyay, A.A., Bosinger, S.E., Maecker, H.T., Khatri, P., Rouphael, N., Peiris, M., Pulendran, B., 2020. Systems biological assessment of immunity to mild versus severe COVID-19 infection in humans. Science 369, 1210-1220. https://doi.org/10.1126/science.abc6261

Ayres, J.S., 2020. A metabolic handbook for the COVID-19 pandemic. Nat. Metab. 2, 572-585. https://doi.org/10.1038/s42255-020-0237-2

Bhome, R., Del Vecchio, F., Lee, G.-H., Bullock, M.D., Primrose, J.N., Sayan, A.E., Mirnezami, A.H., 2018. Exosomal microRNAs (exomiRs): Small molecules with a big role in cancer. Cancer Lett. 420, 228-235. https://doi.org/https://doi.org/10.1016/j.canlet.2018.02.002

Björkman, L., Mårtensson, J., Winther, M., Gabl, M., Holdfeldt, A., Uhrbom, M., Bylund, J., Højgaard Hansen, A., Pandey, S.K., Ulven, T., Forsman, H., Dahlgren, C., 2016. The Neutrophil Response Induced by an 
Agonist for Free Fatty Acid Receptor 2 (GPR43) Is Primed by Tumor Necrosis Factor Alpha and by Receptor Uncoupling from the Cytoskeleton but Attenuated by Tissue Recruitment. Mol. Cell. Biol. 36, 2583-2595. https://doi.org/10.1128/MCB.00161-16

Carneiro, A.M.D., Cook, E.H., Murphy, D.L., Blakely, R.D., 2008. Interactions between integrin alphallbbeta3 and the serotonin transporter regulate serotonin transport and platelet aggregation in mice and humans. J. Clin. Invest. 118, 1544-1552. https://doi.org/10.1172/JCI33374

Chaly, Y., Hostager, B., Smith, S., Hirsch, R., 2014. Follistatin-like protein 1 and its role in inflammation and inflammatory diseases. Immunol. Res. 59, 266-272. https://doi.org/10.1007/s12026-014-8526-z

Cingöz, O., Goff, S.P., 2018. Cyclin-dependent kinase activity is required for type I interferon production. Proc. Natl. Acad. Sci. 115, E2950 LP-E2959. https://doi.org/10.1073/pnas.1720431115

Duerschmied, D., Suidan, G.L., Demers, M., Herr, N., Carbo, C., Brill, A., Cifuni, S.M., Mauler, M., Cicko, S., Bader, M., Idzko, M., Bode, C., Wagner, D.D., 2013. Platelet serotonin promotes the recruitment of neutrophils to sites of acute inflammation in mice. Blood 121, 1008-1015. https://doi.org/10.1182/blood-2012-06-437392

Gao, X., Liu, Y., Zou, S., Liu, P., Zhao, J., Yang, C., Liang, M., Yang, J., 2021. Genome-wide screening of SARS-CoV-2 infection-related genes based on the blood leukocytes sequencing data set of patients with COVID-19. J. Med. Virol. 93, 5544-5554. https://doi.org/https://doi.org/10.1002/jmv.27093

Gleeson, M., Bishop, N.C., Stensel, D.J., Lindley, M.R., Mastana, S.S., Nimmo, M.A., 2011. The antiinflammatory effects of exercise: mechanisms and implications for the prevention and treatment of disease. Nat. Rev. Immunol. 11, 607-615. https://doi.org/10.1038/nri3041

Gomes, C.P.C., Kim, T.-K., Wang, K., He, Y., 2015. The implications on clinical diagnostics of using microRNA-based biomarkers in exercise. Expert Rev. Mol. Diagn. 15, 761-772. https://doi.org/10.1586/14737159.2015.1039517

Gorji-Bahri, G., Hashemi, A., Moghimi, H.R., 2018. ExomiRs: A Novel Strategy in Cancer Diagnosis and Therapy. Curr. Gene Ther. 18, 336-350. https://doi.org/10.2174/1566523218666181017163204

Henry, B.M., Vikse, J., Benoit, S., Favaloro, E.J., Lippi, G., 2020. Hyperinflammation and derangement of renin-angiotensin-aldosterone system in COVID-19: A novel hypothesis for clinically suspected hypercoagulopathy and microvascular immunothrombosis. Clin. Chim. Acta 507, 167-173. https://doi.org/https://doi.org/10.1016/j.cca.2020.04.027

Hers, I., 2007. Insulin-like growth factor-1 potentiates platelet activation via the IRS/PI3Kalpha pathway. Blood 110, 4243-4252. https://doi.org/10.1182/blood-2006-10-050633

Jones, B.A., Beamer, M., Ahmed, S., 2010. Fractalkine/CX3CL1: a potential new target for inflammatory diseases. Mol. Interv. 10, 263-270. https://doi.org/10.1124/mi.10.5.3 
Lévy, Y., Wiedemann, A., Hejblum, B.P., Durand, M., Lefebvre, C., Surénaud, M., Lacabaratz, C., Perreau, M., Foucat, E., Déchenaud, M., Tisserand, P., Blengio, F., Hivert, B., Gauthier, M., Cervantes-Gonzalez, M., Bachelet, D., Laouénan, C., Bouadma, L., Timsit, J.-F., Yazdanpanah, Y., Pantaleo, G., Hocini, H., Thiébaut, R., 2021. CD177, a specific marker of neutrophil activation, is associated with coronavirus disease 2019 severity and death. iScience 24, 102711. https://doi.org/10.1016/j.isci.2021.102711

Li, H., Mou, Q., Li, P., Yang, Z., Wang, Z., Niu, J., Liu, Y., Sun, Z., Lv, S., Zhang, B., Yin, C., 2019. MiR-486-5p inhibits IL-22-induced epithelial-mesenchymal transition of breast cancer cell by repressing Dock1. J. Cancer 10, 4695-4706. https://doi.org/10.7150/jca.30596

Li, X.-B., Zhang, Z.-R., Schluesener, H.J., Xu, S.-Q., 2006. Role of exosomes in immune regulation. J. Cell. Mol. Med. 10, 364-375. https://doi.org/10.1111/j.1582-4934.2006.tb00405.x

Love, M.I., Huber, W., Anders, S., 2014. Moderated estimation of fold change and dispersion for RNA-seq data with DESeq2. Genome Biol. 15, 550. https://doi.org/10.1186/s13059-014-0550-8

Lowder, T., Padgett, D.A., Woods, J.A., 2005. Moderate exercise protects mice from death due to influenza virus. Brain. Behav. Immun. 19, 377-380. https://doi.org/https://doi.org/10.1016/j.bbi.2005.04.002

Minasyan, H., Flachsbart, F., 2019. Blood coagulation: a powerful bactericidal mechanism of human innate immunity. Int. Rev. Immunol. 38, 3-17. https://doi.org/10.1080/08830185.2018.1533009

Nair, V.D., Ge, Y., Li, S., Pincas, H., Jain, N., Seenarine, N., Amper, M.A.S., Goodpaster, B.H., Walsh, M.J., Coen, P.M., Sealfon, S.C., 2020. Sedentary and Trained Older Men Have Distinct Circulating Exosomal microRNA Profiles at Baseline and in Response to Acute Exercise. Front. Physiol. 11, 605.

https://doi.org/10.3389/fphys.2020.00605

Nieman, D.C., Wentz, L.M., 2019. The compelling link between physical activity and the body's defense system. J. Sport Heal. Sci. 8, 201-217. https://doi.org/https://doi.org/10.1016/j.jshs.2018.09.009

Park, J.H., Moon, J.H., Kim, H.J., Kong, M.H., Oh, Y.H., 2020. Sedentary Lifestyle: Overview of Updated Evidence of Potential Health Risks. Korean J. Fam. Med. 41, 365-373.

https://doi.org/10.4082/kjfm.20.0165

Rau, A., Marot, G., Jaffrézic, F., 2014. Differential meta-analysis of RNA-seq data from multiple studies. BMC Bioinformatics 15, 91. https://doi.org/10.1186/1471-2105-15-91

Rivas-Fuentes, S., Valdés, V.J., Espinosa, B., Gorocica-Rosete, P., Salgado-Aguayo, A., 2021. Could SARSCoV-2 blocking of ACE2 in endothelial cells result in upregulation of CX3CL1, promoting thrombosis in COVID-19 patients? Med. Hypotheses 151, 110570. https://doi.org/10.1016/j.mehy.2021.110570

Ryan, P.M., Caplice, N.M., 2020. Is Adipose Tissue a Reservoir for Viral Spread, Immune Activation, and Cytokine Amplification in Coronavirus Disease 2019? Obesity 28, 1191-1194.

https://doi.org/https://doi.org/10.1002/oby.22843

Page 9/15 
Sallis, R., Young, D.R., Tartof, S.Y., Sallis, J.F., Sall, J., Li, Q., Smith, G.N., Cohen, D.A., 2021. Physical inactivity is associated with a higher risk for severe COVID-19 outcomes: a study in 48440 adult patients. Br. J. Sports Med. bjsports-2021-104080. https://doi.org/10.1136/bjsports-2021-104080

Sapp, R.M., Shill, D.D., Roth, S.M., Hagberg, J.M., 2017. Circulating microRNAs in acute and chronic exercise: more than mere biomarkers. J. Appl. Physiol. 122, 702-717.

https://doi.org/10.1152/japplphysiol.00982.2016

Shannon, P., Markiel, A., Ozier, O., S. Baliga, N., T. Wang, J., Ramage, D., Amin, N., Schwikowski, B., Ideker, T., 2003. Cytoscape: A Software Environment for Integrated Models. Genome Res. 13, 2498-2504. https://doi.org/10.1101/gr.1239303.metabolite

Sticht, C., De La Torre, C., Parveen, A., Gretz, N., 2018. miRWalk: An online resource for prediction of microRNA binding sites. PLoS One 13, 1-6. https://doi.org/10.1371/journal.pone.0206239

Tahamtan, A., Teymoori-Rad, M., Nakstad, B., Salimi, V., 2018. Anti-Inflammatory MicroRNAs and Their Potential for Inflammatory Diseases Treatment. Front. Immunol. .

Tang, N., Li, D., Wang, X., Sun, Z., 2020. Abnormal coagulation parameters are associated with poor prognosis in patients with novel coronavirus pneumonia. J. Thromb. Haemost. 18, 844-847. https://doi.org/https://doi.org/10.1111/jth.14768

Valadi, H., Ekström, K., Bossios, A., Sjöstrand, M., Lee, J.J., Lötvall, J.O., 2007. Exosome-mediated transfer of mRNAs and microRNAs is a novel mechanism of genetic exchange between cells. Nat. Cell Biol. 9, 654-659. https://doi.org/10.1038/ncb1596

Vivas, D., Roldán, V., Esteve-Pastor, M.A., Roldán, I., Tello-Montoliu, A., Ruiz-Nodar, J.M., Cosín-Sales, J., Gámez, J.M., Consuegra, L., Ferreiro, J.L., Marín, F., Arrarte, V., Anguita, M., Cequier, Á., Pérez-Villacastín, J., 2020. Recomendaciones sobre el tratamiento antitrombótico durante la pandemia COVID-19.

Posicionamiento del Grupo de Trabajo de Trombosis Cardiovascular de la Sociedad Española de Cardiología. Rev. Española Cardiol. 73, 749-757.

https://doi.org/https://doi.org/10.1016/j.recesp.2020.04.006

Vuorio, A., Kovanen, P.T., 2021. PCSK9 inhibitors for COVID-19: an opportunity to enhance the antiviral action of interferon in patients with hypercholesterolaemia. J. Intern. Med. 289, 749-751. https://doi.org/https://doi.org/10.1111/joim.13210

Warren, K.J., Olson, M.M., Thompson, N.J., Cahill, M.L., Wyatt, T.A., Yoon, K.J., Loiacono, C.M., Kohut, M.L., 2015. Exercise Improves Host Response to Influenza Viral Infection in Obese and Non-Obese Mice through Different Mechanisms. PLoS One 10, 1-27. https://doi.org/10.1371/journal.pone.0129713

Womack, C.J., Nagelkirk, P.R., Coughlin, A.M., 2003. Exercise-Induced Changes in Coagulation and Fibrinolysis in Healthy Populations and Patients with Cardiovascular Disease. Sport. Med. 33, 795-807. 
Yao, Y., Xu, K., Sun, Y., Tian, T., Shen, W., Sun, F., Yuan, W., Wu, H., Chen, G., Yuan, L., Zhang, W., Lu, M., Lei, M., 2020. MiR-215-5p inhibits the inflammation injury in septic H9c2 by regulating ILF3 and LRRFIP1. Int. Immunopharmacol. 78, 106000. https://doi.org/10.1016/j.intimp.2019.106000

Yu, G., Wang, L.-G., Han, Y., He, Q.-Y., 2012. clusterProfiler: an R Package for Comparing Biological Themes Among Gene Clusters. Omi. A J. Integr. Biol. 16, 284-287. https://doi.org/10.1089/omi.2011.0118

Zaid, Y., Puhm, F., Allaeys, I., Naya, A., Oudghiri, M., Khalki, L., Limami, Y., Zaid, N., Sadki, K., Ben El Haj, R., Mahir, W., Belayachi, L., Belefquih, B., Benouda, A., Cheikh, A., Langlois, M.-A., Cherrah, Y., Flamand, L., Guessous, F., Boilard, E., 2020. Platelets Can Associate With SARS-CoV-2 RNA and Are Hyperactivated in COVID-19. Circ. Res. 127, 1404-1418. https://doi.org/10.1161/CIRCRESAHA.120.317703

\section{Figures}



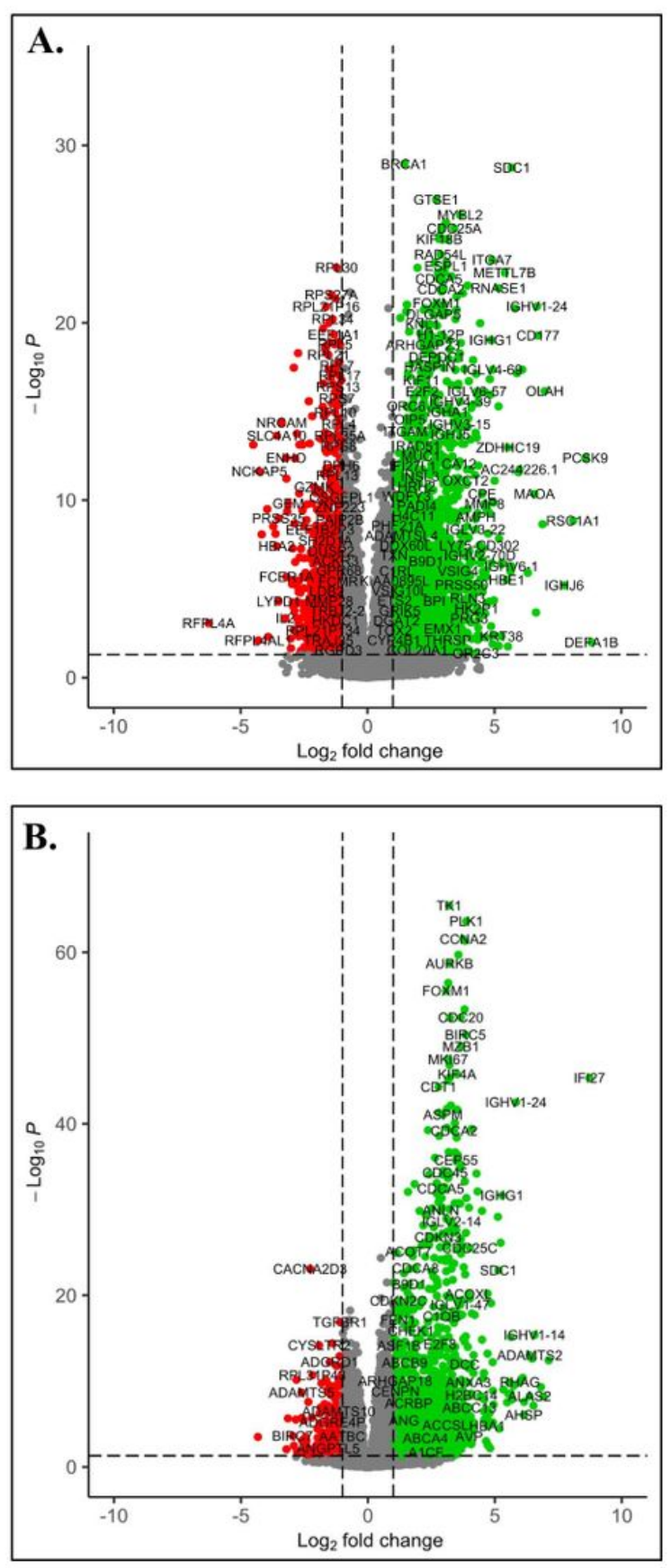

C.

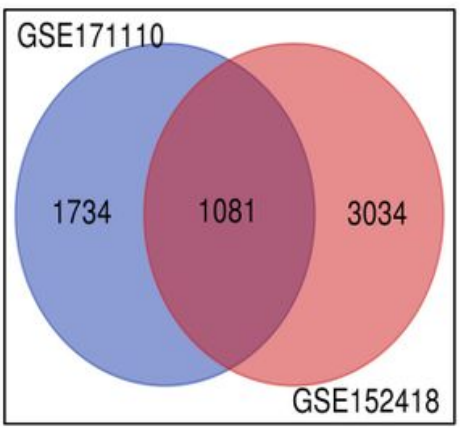

Figure 1

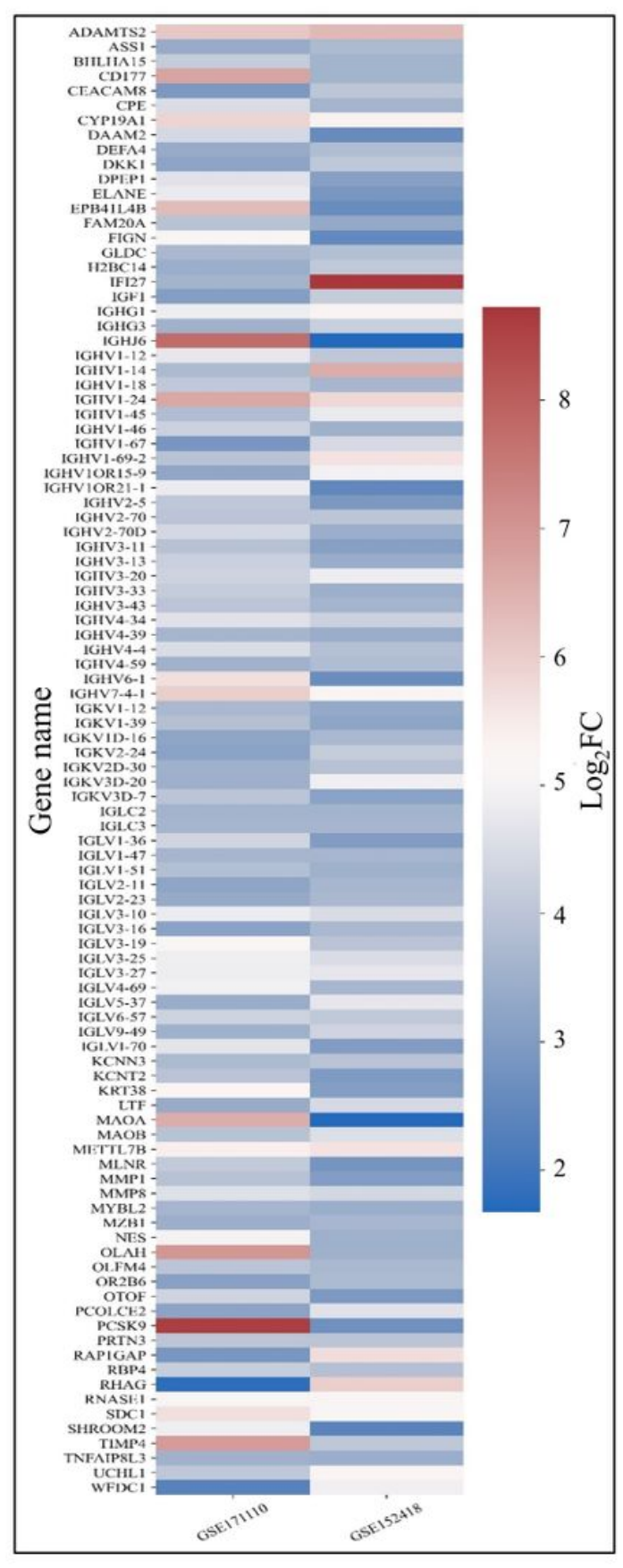

\section{Figure 1}

Screening of upregulated genes in the blood of COVID-19 infected patients. (A.) Volcano plot of DEGs (differentially expressed genes) from GSE152418 (B.) Volcano plot of DEGs (differentially expressed genes) from GSE171110 (C.) Venn diagram representing common upregulated genes in both the datasets (D.) Heatmap showing top 100 upregulated genes. 


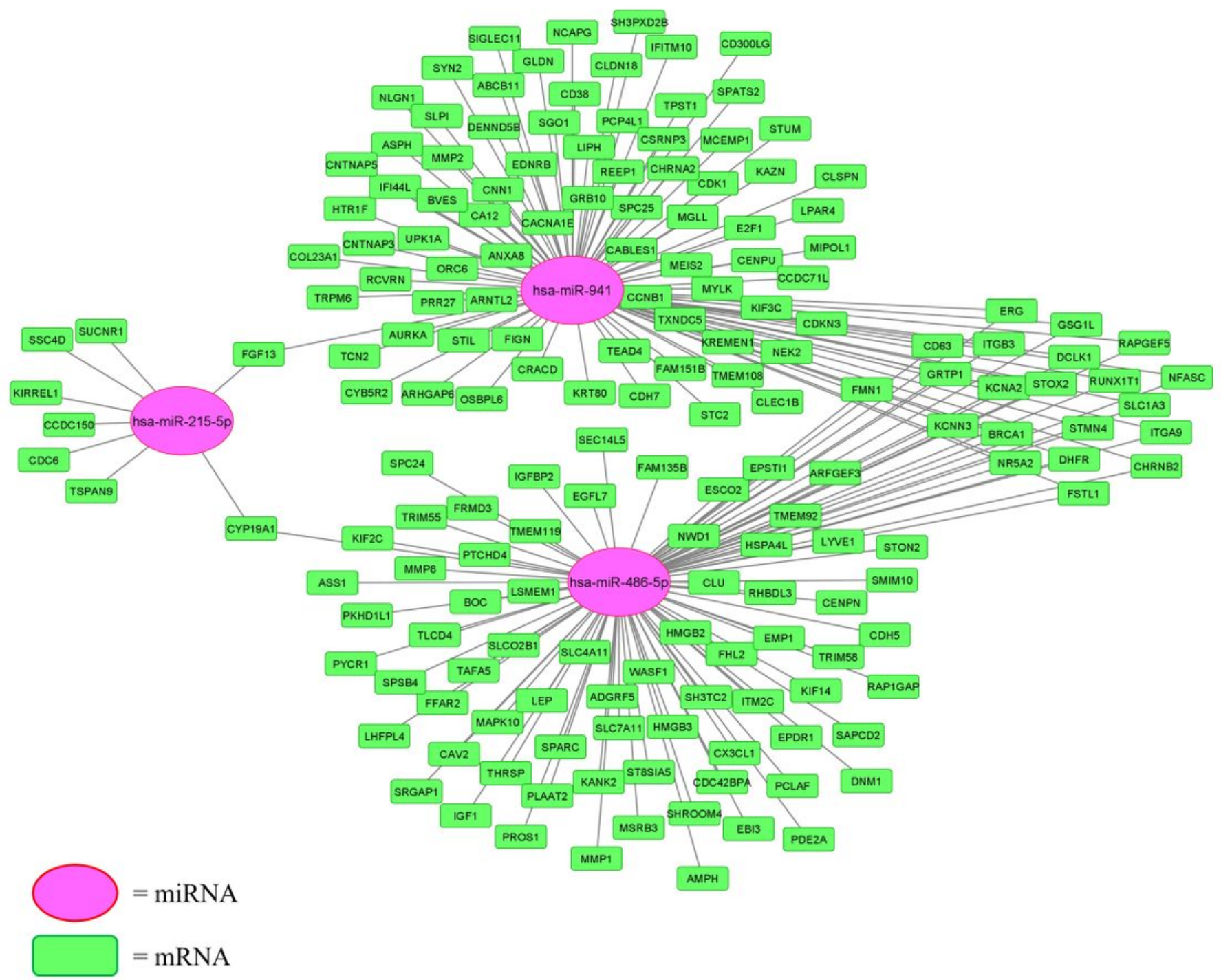

Figure 2

\section{Figure 2}

miRNA-mRNA network in the blood of regularly exercising adults infected with SARS-COV-2 

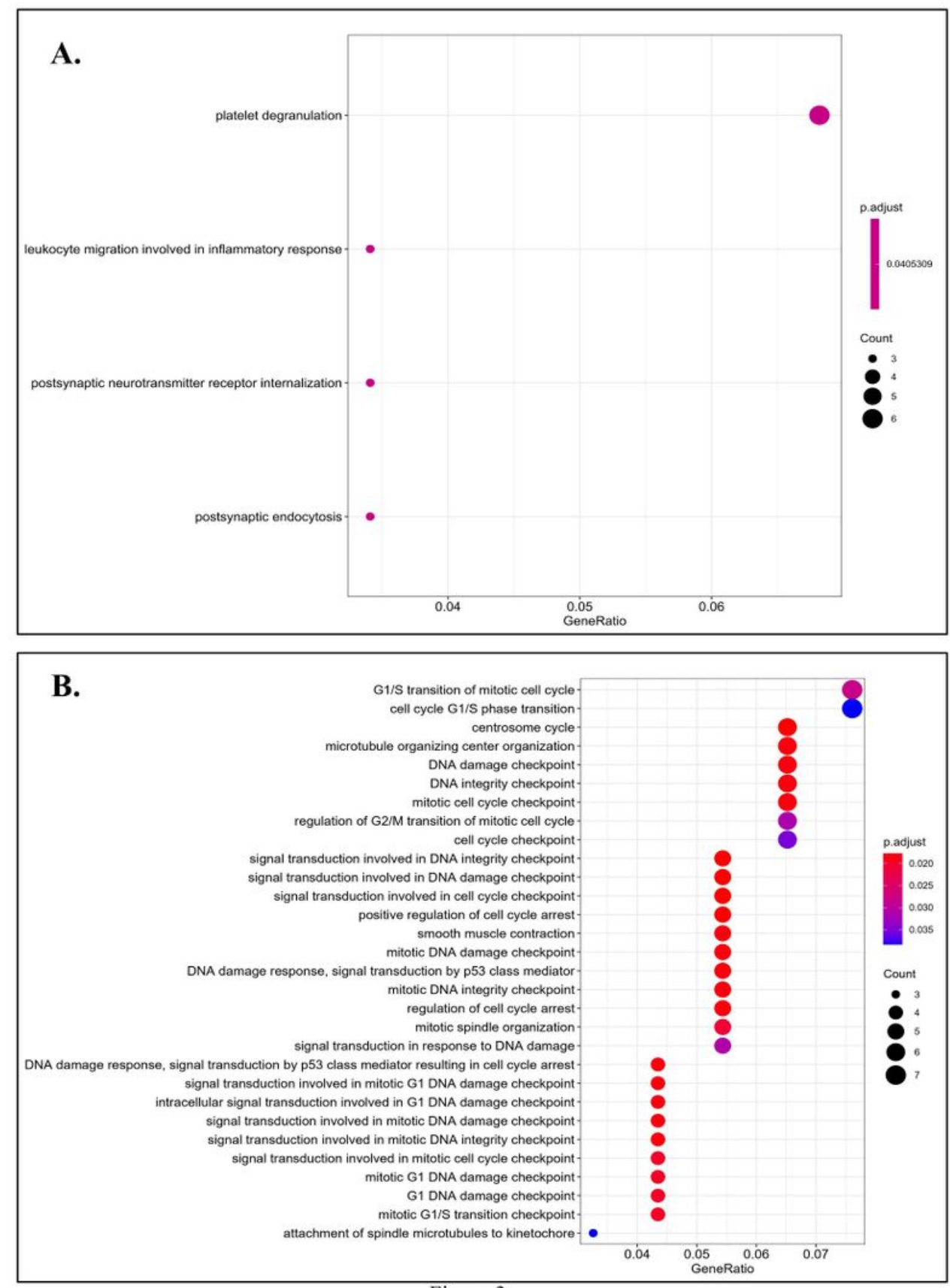

Figure 3

\section{Figure 3}

GO biological process enrichment of mRNAs targeted by the upregulated miRNAs (A.) GO biological process enrichment of mRNAs targeted by hsa-miR-486-5p (B.) GO biological process enrichment of mRNAs targeted by hsa-miR-941. 


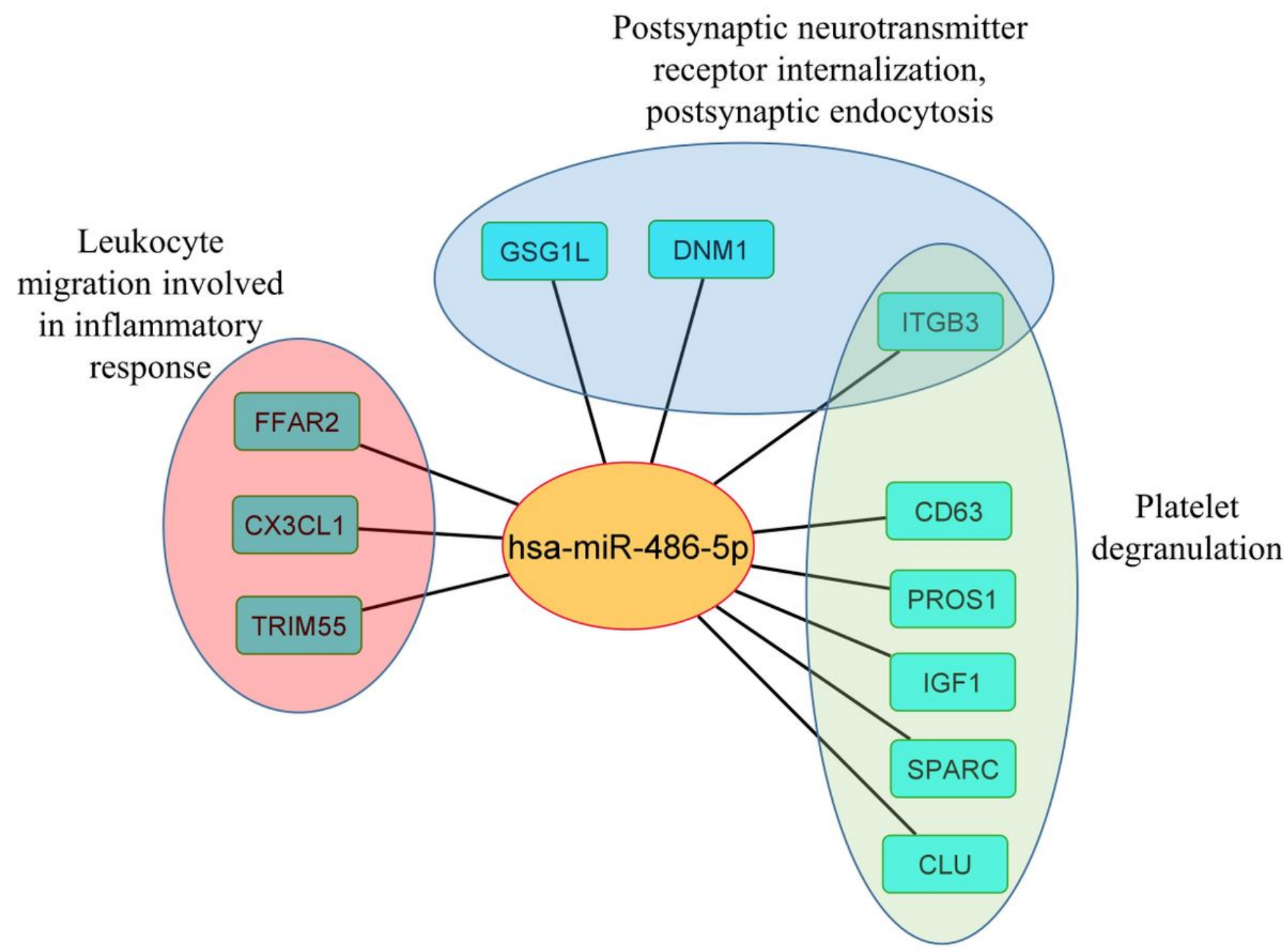

Figure 4

Figure 4

Functional enrichment specific targets of hsa-miR-486-5p

\section{Supplementary Files}

This is a list of supplementary files associated with this preprint. Click to download.

- SupplementaryFileS1.xlsx

- SupplementaryFileS2.xlsx

- floatimage1.jpeg 